\title{
Comparación de dos protocolos para sincronizar ovulación e implementar inseminación artificial en vaquillonas
}

\author{
Callejas, S.; Uslenghi, G.; Catalano, R.; Larghi, J.; Cabodevila, J. \\ Área de Reproducción FISFARVET, Fac. Cs. Vet. UNCPBA, Paraje Arroyo Seco s/n, (7000) Tandil, \\ Argentina. Tel/Fax: 0249-4439850. E-mail: callejas@vet.unicen.edu.ar
}

\begin{abstract}
Resumen
Callejas, S.; Uslenghi, G.; Catalano, R.; Larghi, J.; Cabodevila, J.: Comparación de dos protocolos para sincronizar ovulación e implementar inseminación artificial en vaquillonas. Rev. vet. 25: 2, 100-104, 2014. Se efectuaron dos experimentos (EI y EII) en vaquillonas Bos taurus de 20-24 meses de edad y 3-3,5 de condición corporal (escala 1 a 5). El objetivo fue comparar dos protocolos de sincronización de la ovulación (PSO), utilizando dispositivos intravaginales con progesterona (DISP) combinados con cipionato de estradiol (CPE) administrado al momento de retirar el DISP o benzoato de estradiol (BE) $24 \mathrm{~h}$ más tarde. En el día 0 , las vaquillonas (EI, $\mathrm{n}=16$; EII, $\mathrm{n}=173$ ) recibieron un DISP ( $1 \mathrm{~g}$ de progesterona) más $2 \mathrm{mg}$ BE; en el día 8 se retiró el DISP, se administraron $0,150 \mathrm{mg}$ de D-Cloprostenol y se aplicó, a sendos PSO, una inyección de $1 \mathrm{mg}$ de CPE en dicho momento o $1 \mathrm{mg}$ de BE $24 \mathrm{~h}$ más tarde. En EI, se efectuaron ecografías (días 0,8 y 9 y desde este último momento cada $12 \mathrm{~h}$ hasta el día 11) y muestreos sanguíneos para determinar progesterona plasmática (P4; días 8, 9, 10, 11 y 21); se determinó porcentaje de vaquillonas con cuerpo lúteo (días 0 y 8), los diámetros de los folículos dominante (FD, día 8) y ovulatorio. En EII, se realizó IATF (día 10) y se determinó preñez (día 35). Los análisis estadísticos se realizaron por SAS. Los diámetros del FD $(13,1 \pm 2,4 \mathrm{~mm})$ y ovulatorio $(15,4 \pm 2,2 \mathrm{~mm})$ y el intervalo retiro DISP-ovulación $(65,3 \pm 5,3 \mathrm{~h})$ no difirieron entre PSO ( $p>0,05)$; se observó un efecto del día sobre el nivel de $\mathrm{P} 4(\mathrm{p}<0,05)$, no así del PSO o interacción PSO x día ( $p>0,05)$, el nivel de P4 al día $8(4,6 \pm 4,4 \mathrm{ng} / \mathrm{ml})$ disminuyó a valores $<1 \mathrm{ng} / \mathrm{ml}$ post retiro del DISP. La preñez fue similar entre PSO $(60,7 \% ; \mathrm{p}>0,05)$. Se concluye que en vaquillonas Bos taurus, ambos PSO generan similares cambios ováricos, variaciones en el nivel de P4 y porcentajes de preñez.
\end{abstract}

Palabras clave: vaquillona, sincronización de ovulación, inseminación artificial, comparación de protocolos.

\begin{abstract}
Callejas, S.; Uslenghi, G.; Catalano, R.; Larghi, J.; Cabodevila, J.: Comparison of two protocols to synchronize ovulation and to implement artificial insemination in heifers. Rev. vet. 25: 2, 100-104, 2014. Two experiments were performed in Bos taurus heifers (EI, $\mathrm{n}=16$; EII: 173, body condition score: 3-3.5, scales 1-5, age: 20-24 months). The objective was to compare two protocols for synchronization of ovulation (PSO): progesterone intravaginal devices (PID) combined with estradiol cypionate (ECP) administered at PID removal or estradiol benzoate (EB) $24 \mathrm{~h}$ later. On day 0 , heifers received a PID ( $1 \mathrm{~g}$ of progesterone) plus $2 \mathrm{mg}$ BE; PID was removed on day 8, $0.150 \mathrm{mg}$ of D-cloprostenol were administered and heifers were distributed into two PSO: injection of $1 \mathrm{mg}$ of ECP at that time or $1 \mathrm{mg}$ of EB $24 \mathrm{~h}$ later. In EI, ultrasonography examinations (days 0,8 and 9 and from this last time every $12 \mathrm{~h}$ until day 11) were performed and blood samples were collected to determine plasma progesterone ( $\mathrm{P} 4$, on days: $8,9,10,11$ and 21); percentage of heifers with corpus luteum (days 0 and 8 ), the diameters of the dominant (DF, day 8 ) and ovulatory follicle, were determined. In EII, FTAI (day 10) was performed and pregnancy rate (day 35) was determined. Statistical analysis was performed by SAS. The diameters of DF $(13.1 \pm 2.4 \mathrm{~mm})$ and ovulatory follicle $(15.4 \pm 2.2 \mathrm{~mm})$ and PID removal-ovulation interval $(65.3 \pm 5.3 \mathrm{~h})$ did not differ between PSO $(p>0.05)$. There was an effect of day on the level of P4 $(p<0.05)$, but not of the PSO or PSO by day interaction $(\mathrm{p}>0.05)$, P4 level on day $8(4.6 \pm 4.4 \mathrm{ng} / \mathrm{ml})$ decreased to $<1 \mathrm{ng} / \mathrm{ml}$ after PID removal. Pregnancy rate was similar between PSO $(60.7 \%$; $>0.05)$. In conclusion in
\end{abstract}


Bos taurus heifers, both PSO produce similar ovarian changes, variation in P4 levels and pregnancy rates.

Key words: heifer, synchronization of ovulation, artificial insemination, protocols comparison.

\section{INTRODUCCIÓN}

El control farmacológico de la ovulación mediante la utilización de dispositivos intravaginales con progesterona (DISP) ha permitido implementar programas de inseminación artificial a tiempo fijo (IATF). El protocolo tradicional consiste en la colocación de un DISP más una inyección de benzoato de estradiol (BE) para generar una nueva onda de crecimiento folicular. Siete u ocho días después se retira el dispositivo y se administra un agente luteolítico, $24 \mathrm{~h}$ más tarde se inyecta BE para sincronizar las ovulaciones, realizando la IATF 48 a $56 \mathrm{~h}$ después del retiro del dispositivo ${ }^{9}$.

El protocolo mencionado precedentemente conlleva 4 encierres de los animales para colocar/inyectar las drogas y realizar la IATF. Teniendo en cuenta que esto requiere una mayor logística y que puede ser causa de estrés, se han realizado investigaciones tendientes a reducir a 3 el número de veces que los animales deben pasar por la manga. Para esto, se ha sustituido la segunda administración de BE por cipionato de estradiol (CPE) inyectado en el momento de retirar el dispositivo intravaginal, con resultados dispares ${ }^{3,10}$.

El objetivo del presente trabajo fue aportar información que explique las diferencias observadas, para lo cual se compararon ambos protocolos en vaquillonas, estudiando su efecto sobre las estructuras ováricas, los niveles de progesterona plasmática y el porcentaje de preñez luego de realizar una IATF.

\section{MATERIAL Y MÉTODOS}

Lugar. Los trabajos se realizaron en la Escuela de Educación Agraria $\mathrm{N}^{\circ} 1$ "Dr. Ramón Santamarina", Partido de Tandil (EI) y en un establecimiento agropecuario del Partido de Azul (EII), ambos ubicados en la Provincia de Buenos Aires, Argentina.

Animales, alimentación. Se utilizaron vaquillonas Holando Argentino (EI, $\mathrm{n}=16$ ) y Aberdeen Angus negras (EII, $n=173$ ) de 20-24 meses de edad, las cuales recibieron alimentación pastoril sobre pasturas de Rye Grass, sin suplementación, teniendo libre acceso al agua. La condición corporal (escala 1 a 5; 1: emaciada y 5: obesa) tuvo un rango de 3 a 3,5.

Tratamientos. Se realizaron 2 experimentos: EI y EII. En el día 0, se colocó un dispositivo intravaginal con $1 \mathrm{~g}$ de progesterona (EI: Cronipres 3 usos, Biogénesis Bagó; EII: DIB 1 g, Syntex) más una inyección, intramuscular (im) de $2 \mathrm{mg}$ de BE (Bioestrogen, Biogénesis Bagó). El día 8 se retiró el dispositivo, se ad- ministró 0,150 mg de D-Cloprostenol (Enzaprost D-C, Biogénesis Bagó), y las vaquillonas fueron distribuidas al azar en 2 grupos para recibir los siguientes tratamientos: $1 \mathrm{mg}$ de CPE (ECP estradiol, König) en dicho momento (Grupo CPE, $n=8$ ) o $1 \mathrm{mg}$ de BE a las 24 $h$ posteriores (Grupo BE, $n=8$ ). En el EII, se realizó IATF en el día 10 (50-54 h post retiro del dispositivo), utilizando semen congelado-descongelado proveniente de un toro de probada fertilidad.

Estudios ecográficos. En el día 0, las vaquillonas fueron examinadas mediante ultrasonografía (Chison D 600 VET, China, equipado con un transductor transrectal multifrecuencia de $5 \mathrm{MHz}$ ) para determinar la estructura ovárica predominante (EOp: cuerpo lúteo -CL-, folículos $\geq 10 \mathrm{~mm}$ y folículos $<$ a $10 \mathrm{~mm}$ ). Las ecografías se continuaron en los días 8,9 y a partir de este último momento cada $12 \mathrm{~h}$ hasta el día 11. Estas ecografías se realizaron para determinar el diámetro del folículo dominante (FD) al momento del retiro del dispositivo intravaginal y al momento de ovular; y en el día 8, para registrar si existía un CL en el momento de retirar el dispositivo intravaginal. Se consideró que la vaquillona había ovulado cuando desapareció el FD y en su lugar se encontró un CL en una nueva ecografía realizada en el día 21. En este momento se midió el área del mismo utilizando la función área del equipo de ultrasonografía. La hora de ovulación se determinó como el valor promedio entre la hora en que se encontró el FD y aquélla en la que el mismo había desaparecido. El estudio ecográfico para diagnosticar preñez se efectuó el día 35 post-IATF.

Determinación de progesterona. Se tomaron muestras de sangre por punción de la vena yugular en los días $8,9,10,11$ y 21, para determinar progesterona plasmática (EI) y preñez (EII). Las muestras de sangre se colectaron en tubos heparinizados y fueron centrifugadas (2000 x g durante 15 minutos) dentro de la hora de extraídas. El plasma fue almacenado a $-20^{\circ} \mathrm{C}$ hasta el momento de la medición de progesterona (P4). La determinación de P4 se realizó en la Facultad de Ciencias Veterinarias (UNCPBA, Tandil, Buenos Aires). Se utilizó un kit para RIA (COAT A COUNT, Siemens Healthcare Diagnostic Inc., CA, USA) previamente validado para bovinos ${ }^{14}$. El coeficiente de variación intra-ensayo fue $<7 \%$ para concentraciones entre 0,1 y $40 \mathrm{ng} / \mathrm{ml}$ y el inter-ensayo fue $<3,5 \%$. La sensibilidad fue de $0,01 \mathrm{ng} / \mathrm{ml}$.

Análisis estadístico. En EI se estudió el efecto del protocolo sobre los diámetros del FD en el día 8 y ovu- 
latorio; el intervalo retiro del dispositivo - ovulación, las concentraciones de progesterona y el área del cuerpo lúteo en el día 21. En EII se estudió el efecto del protocolo sobre el porcentaje de preñez a la IATF. Los procedimientos estadísticos utilizados fueron, para las variables discretas, el Proc CATMOD; para las variables continuas, el Proc GLM y para las medidas repetidas en el tiempo, el Proc MIXED. El test de Bartlett se aplicó para determinar homogeneidad de variancia. En el caso del intervalo retiro del dispositivo - ovulación, dado que no se cumplieron los supuestos para realizar un ANOVA, se utilizó el test de Kruskal Wallis. Todos los procedimientos utilizados pertenecieron al paquete estadístico SAS ${ }^{11}$.

\section{RESULTADOS}

Ninguno de los animales perdió el dispositivo intravaginal.

Experimento I. El 68,8\% de las vaquillonas tuvo un $\mathrm{CL}$ en el día 0 y todas las vaquillonas ovularon en respuesta al tratamiento. En la Tabla 1 se describen los cambios ováricos ocurridos desde el día 8 en adelante. El porcentaje de vaquillonas con $\mathrm{CL}$ al retiro del dispositivo $(31,3 \%)$ no difirió entre tratamientos $(\mathrm{p}>0,05)$. En el grupo CPE se produjo una mayor dispersión de las ovulaciones $(p<0,05)$. El área del CL en el día 21 no difirió entre tratamientos como así tampoco el nivel de progesterona en plasma $(6,8 \pm 1,1 \mathrm{ng} / \mathrm{ml}, \mathrm{p}>0,05)$.

No se observaron efectos del protocolo ni de la interacción protocolo $\mathrm{x}$ día $(\mathrm{p}>0,05)$ sobre el nivel de progesterona en plasma; por el contrario, se determinó un efecto del día $(\mathrm{p}<0,05)$. Al retirar el dispositivo intravaginal, el valor promedio de progesterona fue de $4,6 \pm 4,4 \mathrm{ng} / \mathrm{ml}$ descendiendo a niveles inferiores a $1 \mathrm{ng} /$ $\mathrm{ml}$ en los días subsiguientes (Figura 1).

Experimento II. No se observó efecto del protocolo de sincronización de la ovulación sobre el porcentaje de preñez a la IATF ( $p>0,05$; Tabla 2 ).

\section{DISCUSIÓN}

El 100\% de las vaquillonas ovuló en respuesta a la inducción demostrando la eficacia de ambos protocolos. Respuestas similares han sido documentadas con antelación ${ }^{1,13}$. En este experimento no se registraron diferencias entre protocolos en los diámetros del FD en el día 8 y ovulatorio, como tampoco las hubo en el intervalo retiro del dispositivo - ovulación.

El diámetro del folículo ovulatorio $(15,4 \pm 2,3 \mathrm{~mm})$ coincidió con el informado por investigadores que, tra-
Tabla 2. Porcentaje de preñez en vaquillonas IATF según protocolo de sincronización de la ovulación.

\begin{tabular}{lc}
\hline protocolo & $\%$ de preñez \\
\hline CPE & $61,0(50 / 82)$ \\
BE & $60,4(55 / 91)$ \\
\hline
\end{tabular}

CPE: administración de $1 \mathrm{mg}$ de cipionato de estradiol en el momento de retirar el dispositivo intravaginal con progesterona. BE: administración de $1 \mathrm{mg}$ de benzoato de estradiol $24 \mathrm{~h}$ post retiro del dispositivo.

bajando con vaquillonas Holstein, registraron un valor de $14,9 \mathrm{~mm}$ con un rango de 10,8 a $18,5 \mathrm{~mm}^{12}$. En vaquillonas para carne, se ha informado un valor de $15,3 \pm 0,2 \mathrm{~mm}^{5}$ en concordancia con lo anteriormente expresado. Por otro lado, cabe señalar que vaquillonas para leche y carne han tenido similares respuestas ante un tratamiento de control del ciclo estral basado en el uso de dispositivos intravaginales con progesterona ${ }^{2}$, por lo cual los trabajos anteriores son comparables a pesar de tratarse de vaquillonas con diferentes aptitudes productivas.

El intervalo retiro del dispositivo - ovulación $(65,3 \pm 5,3 \mathrm{~h})$ coincidió con el reportado en un trabajo anterior $(66 \mathrm{~h}){ }^{1}$ y fue menor que el registrado en otro ensayo, en el cual resultó de $80,6 \pm 5,0 \mathrm{~h}^{5}$. Un intervalo intermedio $(73,5 \pm 1,0 \mathrm{~h})$ fue informado en otra publicación ${ }^{2}$. Se ha observado que existe una correlación negativa entre el tamaño del folículo dominante

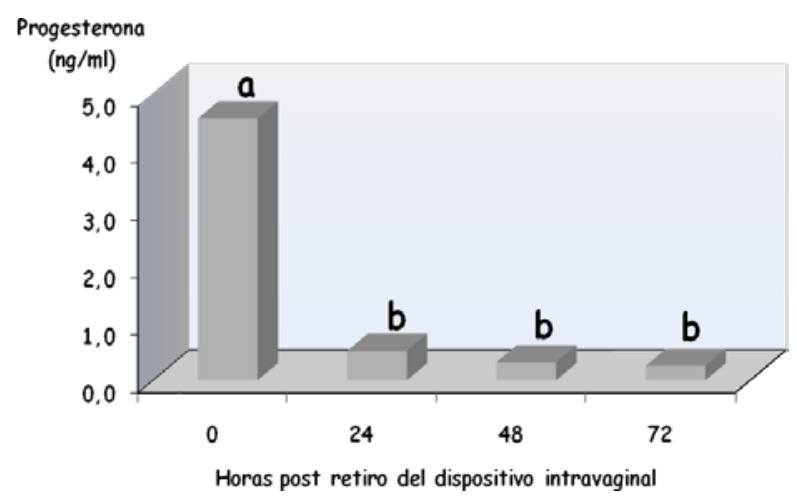

Figura 1. Niveles plasmáticos de progesterona en vaquillonas desde el retiro del dispositivo intravaginal con $1 \mathrm{~g}$ de progesterona (hora 0 ) hasta las 72 horas posteriores. Sobre las columnas, letras distintas indican diferencias significativas 
(posiblemente ovulatorio) presente en el momento de administrar un agente luteolítico y el intervalo a la ovulación ${ }^{4,7}$.

Teniendo en cuenta que en el presente trabajo, el diámetro del folículo dominante determinado en el momento de retirar el dispositivo (equivalente a la administración de un agente luteolítico) fue mayor $(13,1 \pm 2,4$ $\mathrm{mm})$ que el informado por otros autores $(8,6 \pm 0,5 \mathrm{~mm}$ y $10,8 \pm 2,8 \mathrm{~mm})^{2,5}$, se podría hipotetizar que estas diferencias en el diámetro folicular explicarían el mayor intervalo registrado en estos casos. Con respecto al nivel de progesterona, luego de retirado el dispositivo intravaginal se produjo una disminución de los niveles de dicha hormona a valores inferiores a $1 \mathrm{ng} / \mathrm{ml}$, en coincidencia con datos bibliográficos previos ${ }^{2}$.

El área del CL $\left(390,5 \pm 106,1 \mathrm{~mm}^{2}\right)$ fue superior a la registrada en investigaciones anteriores $(303,7 \pm 28,7$ $\left.\mathrm{mm}^{2}\right)^{2}$. En concordancia, el nivel de progesterona en dicho momento fue superior $(6,8 \pm 1,1 \mathrm{ng} / \mathrm{ml})$ al informado en dicho trabajo, de $4,6 \pm 1,0 \mathrm{ng} / \mathrm{ml}$. Se ha determinado que existe relación entre el diámetro del folículo ovulatorio y el tamaño del cuerpo lúteo $\mathrm{y}$, en consecuencia, con el nivel de progesterona ${ }^{15}$. En reportes precedentes se observó un menor diámetro del folículo ovulatorio ${ }^{2}$ en relación al del presente trabajo, lo que podría explicar las diferencias observadas en el área y en los niveles de progesterona, en los respectivos trabajos.

En coincidencia con aseveraciones de otros investigadores, el porcentaje de preñez de los protocolos evaluados fue similar ${ }^{3,10}$. El valor registrado (60,7\%), se encuentra cercano al valor máximo del rango citado en la bibliografía que va de $43,3 \%$ a $62,7 \%{ }^{6}$.

Teniendo en cuenta el rango horario en que se produjeron las ovulaciones en el grupo EI (54-78 h), el tiempo que tarda el espermatozoide en alcanzar el oviducto $(6-8 \mathrm{~h})^{13}$, la vida estimada del ovocito $(8-20 \mathrm{~h})^{8}$ y del espermatozoide $(24-48 \mathrm{~h})^{8}$, el rango horario en que se realizó la IATF (50-54 h) y la condición corporal de las hembras ( $\geq 3$, escala 1 a 5 ), se podría hipotetizar que en el grupo EII se combinaron estos factores de manera óptima lo que permitiría explicar el porcentaje de preñez alcanzado.

No obstante, cabe señalar que estas condiciones no siempre se dan; por ejemplo, en un trabajo publicado sobre distribución de las ovulaciones en los animales que recibieron CPE al retirar el dispositivo intravaginal con progesterona, éstas comenzaron a las $48 \mathrm{~h}$ post retiro y finalizaron a las $96 \mathrm{~h}{ }^{9}$, pudiendo esto influir sobre el resultado a obtener en función de la hora en que se realiza la IATF.

Se concluye que en vaquillonas Bos taurus, el protocolo que utiliza un dispositivo intravaginal con progesterona ( $1 \mathrm{~g}$ ) combinado con un agente luteolítico y sales de estradiol (benzoato al colocarlo y cipionato al retirarlo), genera cambios ováricos y porcentajes de preñez a la IATF similares al protocolo tradicional (administración de BE a 24 h de retirado el dispositivo).
Agradecimiento. A Omar de Dominicis por la colaboración realizada en las actividades de campo (Experimento II).

\section{REFERENCIAS}

1. Ambrose DJ, Rajamahendran RK, Kastelic P, Small JA. 2001. Synchronization of ovulation and conception rates in Holstein heifers given an intravaginal progesterone-releasing device (CIDR), and estradiol cypionate, porcine LH or gonadotropin releasing hormone. Arch Tierz 44: 77-79.

2. Carvalho JB, Carvalho NA, Reis EL, Nichic M, Souza AH, Baruselli PS. 2008. Effect of early luteolysis in progesterone-based timed AI protocols in Bos indicus, Bos indicus $x$ Bos taurus, and Bos taurus heifers. Theriogenology 69: 167-175.

3. Chesta P, Filippi L, Ramos M, Racca D, Bó G. 2009. Evaluación de las tasas de preñez en protocolos de inseminación artificial a tiempo fijo (IATF) utilizando diferentes dosis de cipionato de estradiol en vaquillonas Holando. Anales VII Simposio Internacional de Reproducción Animal IRAC, Córdoba, Argentina, CD n 12.

4. Colazo MG, Martínez MF, Kastelic JP, Mapletoft RJ. 2002. Effects of dose and route of administration of cloprostenol on luteolysis, estrus, and ovulation in beef heifers. Anim Reprod Sci 72: 47-62.

5. Colazo MG, Kastelic JP, Mapletoft RJ. 2003. Effects of estradiol cypionate on ovarian follicular dynamics, synchrony of ovulation, and fertility in CIDR-based, fixedtime AI programs in beef heifers. Theriogenology 60: 855865.

6. Cutaia LE, Peres LC, Pincinato D, Chesta PM, Ramos M, Bo G. 2007. Programas de sincronización de celos en vaquillonas de carne: puntos críticos a tener en cuenta. Anales VII Simp Intern Reprod Anim, Córdoba, Argentina, p. 83-104.

7. Kastelic JP, Ginther OJ. 1991. Factors affecting the origin of the ovulatory follicle in heifers with induced luteolysis. Anim Reprod Sci 26: 13-24.

8. Palma G, Miceli D. 2008. Interacción funcional entre los espermatozoides y el tracto reproductivo de la hembra. En: Biotecnología de la Reproducción (Palma G, ed.), $2^{\circ}$ ed., Edit. Reprobiotec, Mar del Plata, Argentina, p. 93-134.

9. Sá Filho MF; Sales JN; Baruselli P. 2011. Actualización sobre programas de sincronización de celos en ganado bovino de carne. Anales IX Simp Intern Reprod Anim, Córdoba, Argentina, p.165-190.

10. Sales JN, Carvalho JB, Crepaldi GA, Cipriano RS, Jacomini JO, Maio JR, Souza JC, Nogueira GP, Baruselli PS. 2012. Effects of two estradiol esters (benzoate and cypionate) on the induction of synchronized ovulations in Bos indicus cows submitted to a timed artificial insemination protocol. Theriogenology 78: 510-516.

11. SAS. 1989. SAS/STAT ${ }^{\circledR}$ User's Guide, Version 6, vol. 2., 4th ed., SAS Institute Inc., Cary, NC, 846 p.

12. Saumande J, Humblot P. 2005. The variability in the interval between estrus and ovulation in cattle and its determinants. Anim Reprod Sci 8: 171-182. 
13. Scott MA. 2000. A glimpse at sperm function in vivo: sperm transport and epithelial interaction in the female reproductive tract. Anim Reprod Sci 60: 337-348.

14. Toribio RE, Molina JR, Bolaños JM, Kindahl H. 1994. Blood levels of the prostaglandin F2 metabolite during the postpartum period in Bos indicus cows in the humid tropics. J Vet Med A 41: 630-639.
15. Vasconcelos JL, Sartori R, Oliveira HN, Guenther JG, Wiltbank MC. 2001. Reduction in size of the ovulatory follicle reduces subsequent luteal size and pregnancy rate. Theriogenology 56: 307-314.

\section{SCImago Journal \& Country Rank}

\section{Powered by}

\section{Revista Veterinaria aumentó su índice de impacto}

Noticias de Scimago Research Group (Scimago Journals \& Country Ranks, Scopus-Elsevier) comunican que la publicación de nuestra casa, Revista Veterinaria, aumentó su índice de impacto. El índice SJR mide la influencia científica (impacto) del artículo de una revista, expresando cuán importante es el "artículo promedio" de la publicación en la discusión científica global (sistema Thomson Reuters).

Para nuestra revista, tal indicador había sido de 0,03 entre 2008 y 2011, aumentando a 0,05 en 2012 y a 0,11 en 2013, último período evaluado. El aumento fue del 370\%. Asimismo, surge para nuestra publicación un sostenido descenso del indicador que relaciona "citas versus autocitas", demostrando que los autores de los artículos están abandonando la práctica de citar sus propias publicaciones anteriores.

Por último, se advierte que según este portal, nuestra "Revista Veterinaria" continúa siendo la única publicación de esta rama de la ciencia que posee índice de impacto en Argentina. Para el resto del cono sur tal distinción recae en Brasil, Chile, Colombia y Venezuela. No registran índice de impacto las revistas de veterinaria de Bolivia, Paraguay, Uruguay, Perú y Ecuador. En revistas de veterinaria, a nivel mundial el mayor índice de impacto (1,22 puntos) recae en Veterinary Microbiology (Amsterdam, Holanda). 